\title{
O MOSAICO PATOGÊNICO DA PANCREATITE AGUDA GRAVE
}

\author{
THE PATHOGENIC MOSAIC OF SEVERE ACUTE PANCREATITIS
}

\author{
Márcio Cavalcante Carneiro,ACBC-RJ'1 ${ }^{\text {; Rodrigo Siqueira-Batista² }}$
}

\begin{abstract}
RESUMO: A pancreatite aguda tem sido alvo de grandes discussões que vão desde o entendimento de sua fisiopatologia até a investigação de novas modalidades terapêuticas. Reconhece-se que a necrose pancreática e a falência orgânica múltipla - mormente associadas à infecção - representam os principais fatores determinantes da evolução para o óbito, mas, apesar dos grandes avanços em seu estudo, a doença permanece como um desafio para o clínico e o cirurgião. Deste modo, um melhor conhecimento dos mecanismos envolvidos em sua fisiopatologia pode ser a chave para um tratamento mais eficaz, principalmente em relação às formas graves, cuja letalidade ainda encontra-se substancialmente elevada. Baseado nestas considerações, o presente trabalho tem por objetivo a revisão bibliográfica da fisiopatologia, dos fatores patogênicos envolvidos na história natural da pancreatite aguda grave e dos eventos associados à síndrome da resposta inflamatória sistêmica e à sepse, esboçando-se assim o mosaico patogênico desta importante condição (Rev. Col. Bras. Cir. 2004; 31(6): 391-397).
\end{abstract}

Descritores: Pancreatite; Doença aguda; Pancreatite necrosante aguda; Síndrome séptica.

\section{INTRODUÇÃO}

\begin{abstract}
Em moléstias agudas, não é aconselhável prognosticar a morte ou a recuperação.
\end{abstract}

Hipócrates de Cós

Há aproximadamente um século vem sendo dada grande atenção ao entendimento da fisiopatologia, do diagnóstico, da classificação e do tratamento da pancreatite aguda (PA). Obviamente, durante este período, grandes avanços foram obtidos, com base em observações clínicas, estudos experimentais e na criação de novos recursos de investigação. Isto resultou em uma melhor compreensão da moléstia, com conseqüente diminuição das taxas de letalidade.

Apesar da grande evolução nos conhecimentos acerca da PA, muitas interrogações permanecem sem resposta, principalmente em relação à fisiopatologia, visto que há desencadeamento de processos ainda pouco entendidos, os quais convergem para um mecanismo central - independente da etiologia -, caracterizado pela ativação de enzimas pancreáticas, com conseqüente emergência de autodigestão tissular e inflamação local. Todo este mosaico patogênico deságua na cascata de resposta inflamatória sistêmica (SIRS) (Quadro 1) ${ }^{1-3}$. Também não é ainda possível entender o porquê de um certo número de pacientes evoluir com formas graves de doença e outros não.

Com base nestas considerações, o presente trabalho tem por escopo a revisão bibliográfica da fisiopatologia e dos mecanismos relacionados à resposta inflamatória sistêmica e à falência orgânica associadas a pancreatite agu- da grave (PAG), tomando como ponto de partida um breve comentário histórico à moléstia.

\section{AS 'ORIGENS’}

O relato mais antigo de PA deve-se ao cirurgião Francês Ambrose Paré apud4,5, em 1579. A partir de então, numerosas descrições foram realizadas ao longo dos dois séculos seguintes, as quais enfatizaram aspectos da anatomia, histologia e fisiologia do pâncreas, bem como das condições mórbidas que o acometem ${ }^{4}$.

O sistema ductal pancreático foi descrito por Wirsung, em 1641, o qual é responsável pela condução das enzimas pancreáticas na sua forma inativa até o trato gastrintestinal, onde as mesmas são ativadas para exercerem sua função $0^{5,6}$.

Em 1856, Ancelet ${ }^{\text {apud3 }}$ apresentou a descrição patológica da PA, da necrose e do abscesso pancreático. Vinte e seis anos depois, Balser ${ }^{\text {apud } 4,5}$ descreveu a necrose gordurosa peripancreática, questionando a sua relação com a inflamação do pâncreas.

A partir deste momento, relatos clínicos e patológicos das várias formas de PA foram feitos por Senn, em 1886, e Fitz, em 1889 apud3,5. Em 1890, Langerhans ${ }^{\text {apud4 }}$ confirmou a associação da necrose gordurosa com a PA.

Opie ${ }^{\text {apud6 }}$, em 1901, observou que muitos pacientes com PA letal apresentavam, durante a autópsia, cálculo biliar impactado no ducto biliar comum, acreditando que o mesmo fazia a bile migrar para o ducto pancreático, fundamentando a "teoria do canal comum" como um mecanismo na provável gênese da pancreatite.

1. Médico Cirurgião do Serviço de Emergência, Hospital Universitário Clementino Fraga Filho - UFRJ; Médico do Serviço de Cirurgia Geral, Hospital Municipal Souza Aguiar - (SMS-RJ); Mestre em Medicina, Universidade Federal do Rio de Janeiro (UFRJ).

2. Professor Titular de Clínica Médica, Faculdade de Medicina de Teresópolis, Fundação Educacional Serra dos Órgãos (FESO); Professor Titular de Filosofia, Curso de Graduação em Ciência da Computação - FESO; Coordenador do Núcleo de Estudos em Filosofia e Saúde (NEFISA) - FESO; Médico da Comissão de Bioética e do Serviço de Clínica Médica, Hospital Universitário Clementino Fraga Filho - UFRJ; Mestre em Medicina pela Universidade Federal do Rio de Janeiro (UFRJ); Mestre em Filosofia pela Pontifícia Universidade Católica do Rio de Janeiro (PUC-Rio); Doutorando em Ciências, Fundação Oswaldo Cruz (FIOCRUZ). 


\begin{tabular}{ll}
\hline Termo & Conceito \\
\hline SIRS & Síndrome de Resposta Inflamatória Sistêmica é uma resposta inespecífica do organismo a várias \\
& agressões $3 / 4$ infecção, queimaduras, pancreatite aguda, trauma e outras. São necessárias duas \\
das seguintes condições: & Temperatura $>38,0^{\circ} \mathrm{C}$ ou $<36,0^{\circ} \mathrm{C}$ \\
& Frequência cardíaca $>90 \mathrm{bpm}$ \\
& Freqüência respiratória $>20$ irpm ou $\mathrm{PaCO}_{2}<32 \mathrm{mmHg}$ \\
& Leucócitos $>12.000 / \mathrm{mm}^{3}$ ou $<4.000 / \mathrm{mm}^{3}$ ou $>10 \%$ de bastões \\
& SIRS desencadeada por infecção bacteriana, viral, fúngica ou parasitária. \\
Sepse & Pressão arterial sistólica $<90 \mathrm{mmHg}$ ou uma redução de $40 \mathrm{mmHg}$ da pressão "basal”. \\
Hipotensão & Aquela associada com disfunção orgânica, hipoperfusão tissular ou hipotensão arterial. \\
Chopue grave & Hipotensão / hipoperfusão tecidual (que, entre outros, inclui acidose láctica, oligúria e disfunção \\
& do nível de consciência) ocasionadas por sepse. Pode ser precoce quando dura menos de uma \\
& hora (por resposta ao tratamento preconizado) ou refratário, com duração maior que uma hora \\
& (após $500 \mathrm{ml}$ soro fisiológico em 30 minutos e uso de aminas vasoativas).
\end{tabular}

Disfunção orgânica múltipla Alterações da função de órgãos de um enfermo grave, de modo que a homeostase não pode ser mantida sem intervenção terapêutica.

Quadro 1 - Definições úteis ao entendimento da síndrome de resposta inflamatória sistêmica (SIRS), da sepse e de suas complicações.

Outro ponto que mereceu atenção dos estudiosos foi a classificação da pancreatite. Em um primeiro momento, os agrupamentos foram baseados em achados cirúrgicos ou autópsias, como disposto por Lagerlof, em 1942, e por Blumenthal, em 1959 apud3. Estes conceitos foram firmados em 1965, no primeiro simpósio de Marseille. Neste evento ficou definido que a PA era subdividida em aguda e recorrente, porém, clinicamente, esta classificação mostrou-se pouco prática, pois dependia do tecido para avaliação histológica, não sendo possível a demarcação daqueles eventos que não eram submetidos à cirurgia, ou naqueles em que não se realizava a autópsia - empregada nos casos de evolução fatal. ${ }^{4}$

A tentativa de classificar clinicamente o paciente com PA e o reconhecimento de sua potencial gravidade foi enfatizado por Ranson ${ }^{7}$, em 1974. Em 1983 define-se a classificação de Cambridge que dividia a pancreatite em leve e grave, caracterizando-se esta última pela existência de falência multissistêmica ou complicações locais (precoces ou tardias). ${ }^{4}$ No ano seguinte ocorreu o segundo simpósio de Marseille (1984), no qual foi estabelecida uma classificação a partir de tecidos obtidos em cirurgia, estratificando a PA em necrótica, hemorrágica ou pseudocisto, sem que tivessem sido definidos, todavia, estes conceitos. ${ }^{3} \mathrm{Na}$ década de 80, Büchler et al ${ }^{8}$, estabeleceram que a necrose pancreática era o principal determinante da gravidade clínica e da mortalidade.

Claramente, a desvantagem dos sistemas baseados nos achados patológicos está na necessidade de tecido pancreático e avaliação histológica para confirmação, o que não é uma situação freqüente na prática clínica. Em contraste, os sistemas baseados em aspectos clínicos não necessitam de confirmação histológica, sendo de mais fácil aplicabilidade no dia a dia dos profissionais que assistem estes pacientes.

Com isto em mente, Beger ${ }^{9}$ propôs, em 1991, um esquema de classificação morfológica e bacteriológica que dependia da realização da tomografia computadorizada para definir os aspectos morfológicos, além da punção por agulha fina para definir a classificação bacteriológica como infectada ou não.

Desta forma, segundo Beger, a PA era divida em: intersticial (edematosa); necrosante (que poderia ser estéril ou infectada); abscesso, e finalmente pseudocisto. Esta classificação ficou conhecida como classificação de Ulm para $\mathrm{PA}^{9}$.

Em 1992, um consenso entre os principais estudiosos no assunto - durante o simpósio de Atlanta - definiu que a PA leve era a forma da doença caracterizada por edema intersticial do pâncreas, eventualmente com áreas microscópicas de necrose parenquimatosa associada ou não à necrose gordurosa peripancreática, podendo ser acompanhado por disfunção orgânica mínima, com recuperação entre 48 e 72 horas ${ }^{4}$. Na PAG, há o desenvolvimento de necrose pancreática que é expressa clinicamente através de falência orgânica - ainda que disfunções orgânicas possam estar presentes ainda no início do processo, antes mesmo do estabelecimento da necrose ${ }^{3,4}$. Outras complicações locais podem estar presentes como o abscesso pancreático ou pseudocisto. As alterações clínicas decorrentes da necrose pancreática são caracterizadas pela presença de três ou mais critérios de Ranson ou oito ou mais pontos pelo escore APACHE II (Acute Physiology and Chronic Health Evaluation $)^{2}$.

Desta forma, quando se fala em PA leve ou grave, a delimitação se refere aos critérios de Atlanta, os quais também tem o papel de unificar a linguagem acerca desta complexa doença.

\section{A CAIXA DE PANDORA: PATOGENIA E FISIOPATOLOGIA}

Por suas implicações clínico-cirúrgicas e pelo grande potencial de morbidade, será dada primazia, nesta discussão, 


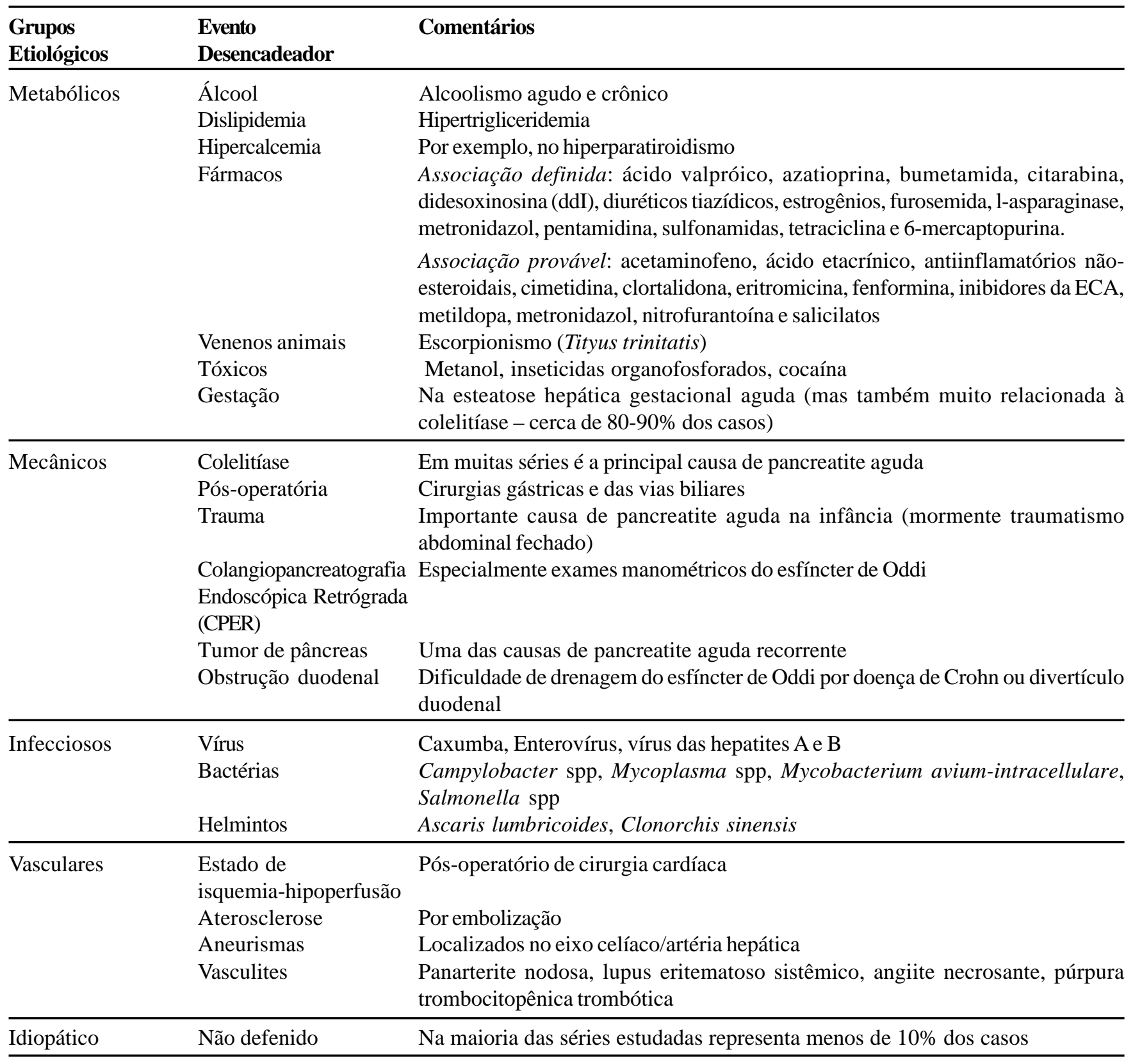

Quadro 2 - Etiologias da pancreatite aguda.

à PAG, condição marcada pelo desenvolvimento de necrose pancreática, cuja expressão clínica mais relevante é a falência orgânica múltipla ${ }^{10,11}$.

Um fato bem estabelecido na patogênese da PA é a existência de um deflagrador - a exposição a um fator causal o qual desencadeia uma série de eventos que culminam com a inflamação do pâncreas. Os principais fatores sabidamente envolvidos no desencadeamento da PA incluem cálculo biliar, ingestão de álcool, medicamentos, toxinas (veneno de escorpião), trauma, colangiopancreatografia endoscópica retrógrada (CPER), cirurgia biliar e gástrica, infecções (vírus, Mycobacterium tuberculosis, helmintos), estenose ou disfunção do esfíncter de Oddi, tumores que obstruem o ducto pancreático, pré-disposição familiar e má formação (pâncreas divisum) (Quadro 2) 3,4,12-18.
Apesar deste reconhecimento causal, uma questão permanece não respondida: como fatores tão diferentes podem confluir para uma via comum? O estado atual da arte está longe de permitir respostas seguras acerca deste ponto. Logo, os mecanismos que estes deflagradores evocam na gênese da PA, não passam de hipóteses, em busca de confirmação.

A idéia mais aceita estabelece que, após a ocorrência do episódio desencadeador, uma série de eventos ocorrem para que haja a inflamação do pâncreas ${ }^{19}$. Estes podem ser divididos em fases precoce e tardia. Num primeiro momento surgem alterações na célula acinar, as quais culminam com ativação precoce do zimogênio localizado intracelularmente. Acredita-se, ainda, que não basta apenas a ativação enzimática, mas também a retenção de enzimas ativadas - dificultando a liberação destas - decorrente de alterações no citoesqueleto 
apical da célula acinar ${ }^{2,3,9,20}$. Os eventos celulares ocorrem minutos após a exposição ao fator causal, tendo sido demonstrado que os peptídeos da ativação do tripsinogênio são gerados num compartimento distinto, dentro da célula, e precocemente no início da pancreatite $3,16,21-24$.

A lesão celular pelas enzimas ativadas aciona a liberação de substâncias (citocinas) que ativam o sistema complemento e as plaquetas, além de atraírem neutrófilos e propiciarem a formação de radicais livres de oxigênio, iniciando assim um processo inflamatório local, o qual leva à alteração de permeabilidade capilar, contribuindo para formação de edema $^{25,26}$. Uma vez na circulação sistêmica, estas substâncias desencadeiam uma resposta inflamatória global em questão de horas - pela amplificação da resposta inflamatória inicial - havendo, então, agravamento da inflamação pancreática e dos tecidos adjacentes, além do surgimento de manifestações como taquicardia, febre e hipotensão ${ }^{20,27,28}$.

Uma série de células e de moléculas participam do intrincado processo inflamatório na PA grave ${ }^{29,30}$. Evidências clínicas e experimentais tem demonstrado que as citocinas pró-inflamatórias e o estresse oxidativo estão criticamente implicados na patogênese da PA grave ${ }^{31}$. Os níveis de interleucina- $1 \beta$ (IL-1 $\beta$ ), e de fator de necrose tumoral alfa (TNF- $\alpha$ ) - e do seu RNA correspondente - se elevam durante a evolução da PA, podendo representar importantes mediadores na propagação da SIRS ${ }^{29,31,32}$. O TNF- $\alpha$ estimula leucócitos e o endotélio vascular a liberar outras citocinas (bem como mais TNF- $\alpha$ ), a expressar moléculas de adesão na superfície celular e a aumentar o turnover de ácido aracdônico. Embora o TNF- $\alpha$ seja um mediador central na patogênese da SIRS (promovendo febre, anorexia, choque, extravasamento capilar, intensificação da citotoxicidade dos leucócitos e da função das células natural killer, entre outros efeitos) é apenas uma das várias citocinas que contribuem para o processo, onde outros mediadores também têm um papel fundamental. Ademais, moléculas como IL-6, IL-8, IL-12, a proteína quimiotática de monócitos 1 (MCP-1), o fragmento C5a do sistema complemento, ICAM-1 (molécula de aderência intercelular 1), a substância P e o PAF (fator ativador de plaquetas), também se encontram em níveis séricos mais altos na PA grave, concorrendo para (1) liberação de histamina (por basófilos e mastócitos), (2) indução da síntese de proteínas de fase aguda, (3) quimiotaxia de monócitos e neutrófilos, (4) adesão destas últimas células ao endotélio, (5) ativação de monócitos e macrófagos - neste âmbito há participação do NF-kappa-B (fator nuclear kappa-B), enquanto mediador da transcrição de inúmeras citocinas, como o demonstrado em algumas investigações - e (6) estimulação da angiogênese, entre outros efeitos biológicos $22,24,33-36$.

A despeito do estado de inflamação expresso nestes perfis de citocinas, estudos tem demonstrado a ocorrência de um certo grau de imunossupressão nos enfermos com PA, a qual parece estar implicada - juntamente com outros fatores — na maior susceptibilidade às infecções e sepse ${ }^{37}$.

$O$ processo inflamatório gera alteração da permeabilidade capilar, evoluindo com edema tissular além de alterações na microcirculação pancreática, levando a hipóxia tissular, isquemia e necrose inicialmente da gordura interlobular e peripancreática. Há evolução, posteriormente, para lesão e destruição tissular nos lóbulos pancreáticos periféricos e dos ductos pancreáticos, caracterizando a necrose pancreática, que pode envolver também vasos e gerar hemorragia ou trombose $^{20}$. Uma vez instalada a necrose pancreática, a repercussão clínica é evidente proporcional à extensão do parênquima pancreático acometido ${ }^{38}$.

Durante a evolução do episódio de pancreatite necrótica, alguns caminhos podem ser seguidos. A primeira opção, e talvez a mais desejada, é a liquefação e reabsorção da área necrótica com resolução espontânea do quadro. No entanto, com as melhorias das condições de cuidados intensivos e controle das insuficiências orgânicas, os casos de PA necrótica que não se resolvem espontaneamente podem evoluir com infecção em torno da segunda para terceira semana de doença, acreditando-se que a origem desta infecção seja pela translocação bacteriana a partir do trato gastrintestinal, ${ }^{39}$ em geral por bactérias gram-negativas, as quais são capazes de "alimentar" a resposta inflamatória sistêmica a partir da liberação de endotoxinas. Esta situação caracteriza a necrose infectada, a qual pode ser confirmada pela punção com agulha fina ou pela presença de gás - visualizável na tomografia computadorizada de abdome -, produzido pela ação de diferentes patógenos ${ }^{12}$.

Uma vez instalada a necrose pancreática, costumase encontrar um paciente grave, no qual a presença de SIRS é evidente, podendo esta ser ainda complicada com infecção. $\mathrm{O}$ agravamento progressivo do quadro inflamatório em decorrência do processo infeccioso - muitas vezes estabelecido em sepse - torna o prognóstico sombrio, levando à disfunção orgânica múltipla (MODS), com letalidade marcadamente mais elevada ${ }^{2,3,30-33}$.

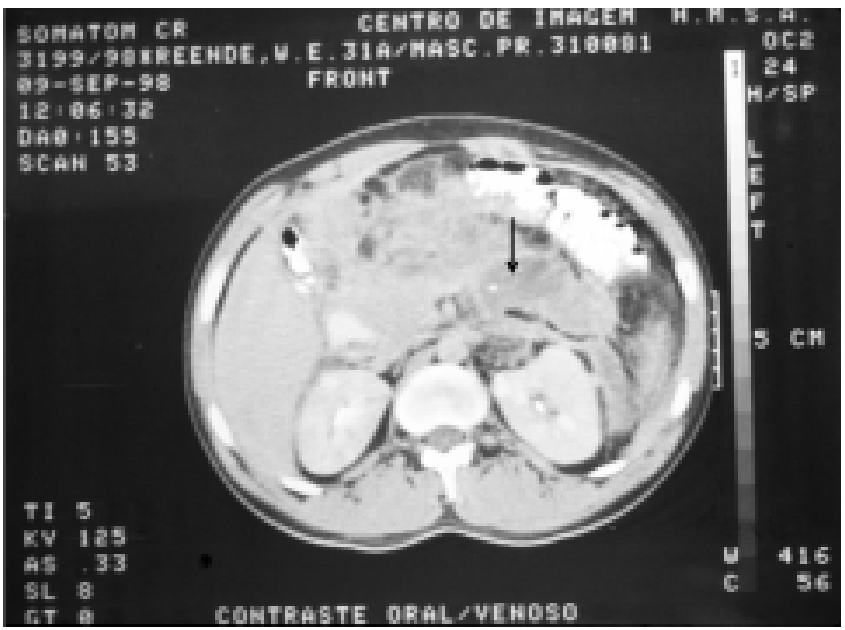

Figura 1 - Tomografia Computadorizada de abdome com contraste oral e venoso, evidenciando área heterogênia em topografia de pâncreas, correspondendo a necrose pancreática (seta branca) e coleção (seta preta).

\section{ÀS PORTAS DO HADES: O PAPEL DA SEPSE}

A infecção pancreática, peripancreática e o abscesso pancreático surgem, muito freqüentemente, no decorrer da PA grave (Figura 1). A ocorrência ou não de infecção depende 
principalmente da extensão da necrose, do grau de isquemia e hipoperfusão do órgão e da presença de insuficiência de um ou vários órgãos ${ }^{1}$. Nestes casos, há elevação da letalidade para mais de $80 \%$, conforme algumas séries, pelo agravamento da SIRS conseqüente à sepse ${ }^{40,41}$.

Os principais agentes implicados na infecção incluem Escherichia coli, Klebsiella pneumoniae, Staphylococcus aureus, Pseudomonas aeruginosa e Proteus mirabilis, sendo descritas, nos últimos anos, ocorrências por Enterococcus faecalis, Enterobacter aerogenes, Bacteroides fragilis, Candida spp, Serratia marcences e Serratia putrefaciens ${ }^{40}$.

Em relação a estes patógenos, um grande número de substâncias e subprodutos celulares podem corroborar para a perpetuação da SIRS, no âmbito de uma resposta séptica. Entre estas destacam-se as exotoxinas - como a toxina A produzida por $P$. aeruginosa e a toxina- 1 da síndrome do choque tóxico (TSST-1) sintetizada e liberada por S. aureus —, os polissacarídeos de superfície de Candida albicans e o ácido lipoteicóico de $S$. aureus. Todavia, as mais potentes e melhor estudadas são as endotoxinas de bactérias gram-negativas, que derivam da parede celular e são formadas principalmente por lipopolissacarídeos (LPS) ${ }^{42,43}$.

Uma proteína plasmática ligadora de lipopolissacarídeos, a LBP, transfere os LPS aos $\mathrm{CD}_{14}$ na superfície de monócitos, macrófagos e neutrófilos, desencadeando rapidamente a produção e liberação de citocinas, tais como o TNF-a - que possui, como se viu, relevante papel na PA grave - as quais amplificam o sinal dos LPS e o transmitem para outras células e tecidos. Este mediador e a IL-1 apresentam interação frequiente, amplificando suas respostas e agindo de maneira sinérgica, produzindo vários efeitos biológicos, tais como depressão miocárdica — ainda que este não seja o único mecanismo descrito para este evento — resultando em uma baixa fração de ejeção, o que acaba por concorrer para acentuação da isquemia tissular ${ }^{42,43}$.

As endotoxinas também podem ativar a cascata do complemento, usualmente pela via alternativa, resultando na liberação de C3 e C5, os quais induzem vasodilatação, aumento da permeabilidade vascular, agregação plaquetária e ativação e agregação de neutrófilos. Esses mediadores, podem ser, em parte, responsáveis pelas alterações microvasculares presentes na sepse grave e no choque séptico. As endotoxinas, também levam à liberação de bradicinina (pela ativação do fator XII 3/4 fator de Hageman), calicreína e cininogênio. A bradicinina é um potente agente vasodilatador e hipotensor, enquanto a ativação do fator XII, pode levar a ativação da via intrínseca da coagulação, resultando em con- sumo dos seus fatores e coagulação intravascular disseminada $(\mathrm{CID})^{42}$.

Este estado de inflamação sistêmica - já presente no próprio quadro da PA grave, mas agravado pela infecção (e sepse) - concorre para um aprofundamento do componente pró-inflamatório da resposta (TNF- $\alpha$, TNF- $\beta$, IL-1, IL-2, IL-6, IL-8, IL-12, interferon , fator estimulador de colônias de granulócitos e monócitos), gerando alterações como disfunção miocárdica, vasodilatação, diminuição da resistência vascular periférica, lesão endotelial disseminada - com extravasamento de fluidos e conseqüente edema intersticial e hipovolemia -, distúrbios da coagulação com formação de microtrombos, que diminuem o aporte de oxigênio e nutrientes para os tecidos afetados. Associado a isso, é observada maior liberação de hormônios contra-insulínicos (glucagon, corticosteróides, catecolaminas e hormônio do crescimento), resultando em hipermetabolismo, com aumento da glicogenólise e da gliconeogênese hepática, aumento da lipólise, diminuição da lipogênese e do catabolismo protéico muscular, intestinal e do tecido conjuntivo. Todos esses mecanismos, em conjunto, levam à hipóxia, acidose láctica - a hiperlactatemia associa-se com a gravidade da doença e morte celular - tornando ainda mais propícia a evolução para choque refratário, disfunção de múltiplos órgãos e morte.

\section{PONDERAÇÕES FINAIS}

A pancreatite aguda permanece como uma importante condição clinico-cirúrgica na medicina atual, tanto por sua freqüência, quanto pela potencial gravidade. Em relação à patogênese, muitos pontos permanecem em aberto, à espera de que novas investigações possam trazer maior luz aos mecanismos implicados, do desencadeamento da inflamação pancreática à síndrome de resposta inflamatória sistêmica instalada.

O grande desafio que esta melhor compreensão fisiopatológica impõe é, justamente, minimizar os riscos de que os quadros extremamente graves - que podem sobrevir -, levem o paciente à inviabilidade biológica, algo já demarcado pelo próprio Hipócrates apud44.

Em febres contínuas, se as áreas externas do corpo estiverem frias mas os órgãos internos muito quentes, e se a pessoa tiver sede, esse é um sintoma mortal ${ }^{45}$.

Eis um repto que atravessa o tempo, devendo evocar todos os esforços para sua suplantação, quiçá como o sonhado em outrora pelo grande médico de Cós...

\footnotetext{
ABSTRACT

Acute pancreatitis has been the objective of discussion, principally regarding its physiopathology and treatment. It is recognized that pancreatic necrosis, infections and multiple organ dysfunction are associated with high mortality. This disease remains a challenge for physicians and surgeons. However, a better understanding of evolved mechanisms may be the key for an appropriated treatment, principally for severe forms of disease in which mortality remains elevated. This study reviews the physiopathology and natural history of severe acute pancreatitis, and its association to SIRS and sepsis.
}

Key words: Pancreatitis; Acute disease; Pancreatits, Acute necrotizing; Sepsis syndrome 


\section{REFERÊNCIAS}

1. Greenberger NJ, Toskes PP - "Pancreatite aguda e crônica". In Braunwald E, Fauci AS, Kasper DL, et al. - Harrison Medicina Interna. Tradução de Marcio Moacyr Vasconcelos. 15a Edição. Rio de Janeiro. McGraw Hill, 2002, pp.1899-1911.

2. Young LS - "Sepsis syndrome". In Mandell G, Bennet JE, Dolin R - Principles and Practice of Infectious Diseases. $5^{\text {th }}$ Edition. Philadelphia. Churchill Livingstone, 2000, pp. 806817.

3. Carneiro MC. O valor da avaliação clínica e laboratorial inicial simplificada no prognóstico da pancreatite aguda. Dissertação (Mestrado). Rio de Janeiro. Universidade Federal do Rio de Janeiro, 2003, 70p.

4. Bradley EL - "The necessity for a clinical classification of acute pancreatitis the Atlanta system". In Bradley EL - Acute pancreatitis diagnosis and therapy. New York. Raven Press, 1994, pp. 27-32.

5. Chavez Rossell M - História del páncreas y la evolución de los conceptos y clasificación de la pancreatitis. Rev Gastroenterol Peru, 2002, 22(3):243-247.

6. Steer MC - "Pathophysiology and pathogenesis of acute pancreatitis". In Bradley EL - Acute pancreatitis diagnosis and therapy. New York. Raven Press, 1994, pp. 3-11.

7. Ranson JH, Rifkind KM, Roses DF, et al. - Objective early identification of severe acute pancreatitis. Am J Gastroenterol,1974, 61(6):443-451.

8. Büchler M, Uhl W, Malfertheiner P - "Biochemical stoging of acute pancreatitis. In Beger HG, Büchler M - Acute pancreatitis. Berlin. Springer-Verlag, 1987, pp. 143-153.

9. Beger HG - Surgery in acute pancreatitis. Hepatogastroenterology, 1991, 38(2):92-96.

10. Becker V - "Acute pancreatitis: a brief introduction of the pathology". In Beger HG, Büchler M - Acute pancreatitis. Berlin. Springer-Verlag, 1987, pp.3-11.

11. Steer ML, Meldolesi J - Pathogenesis of acute pancreatitis. Annu Rev Med, 1988, 39:95-105.

12. Balthazar EJ - CT diagnosis and staging of acute pancreatitis. Radiol Clin North Am, 1989, 27(1):19-37.

13. Andersen DK - "Pâncreas". In Sabiston DCJ, Lyerly HK Fundamentos de cirurgia. $2^{\text {a }}$ Edição. Rio de Janeiro. Guanabara Koogan, 1996, pp. 345-361.

14. Yeo CJ, Cameron JL - "Pâncreas". In Sabiston DCJ, Lyerly HK - Cirurgia. As bases biológicas da prática cirúrgica moderna.

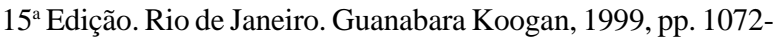
1103.

15. Vital ML, Gomes AP, Gonçalves MLC, et al. "Escorpionismo". In Siqueira Batista R, Gomes AP, Santos SS, et al. (eds) - Manual de infectologia. Rio de Janeiro. Revinter; 2003, pp. 503-505.

16. Chebli JM, de Souza AF, de Paulo GA, et al. - Pancreatite hiperlipidêmica: curso clinico. Arq Gastroenterol, 1999, 36(1): 4-9.

17. Chebli, JMF, Ferrari AP, Silva MRR, et al. - Microcristais biliares na pancreatite aguda idiopática: indício para etiologia biliar oculta subjacente. Arq Gastroenterol, 2000, 7(2):93-101.

18. Farhoud S, Stephani SM, Bromberg SH - Pancreatite aguda devida a hematoma intramural do duodeno por uso de anticoagulante. Arq Gastroenterol, 2001, 38(1): 53-56.

19. Steer ML - Classification and pathogenesis of pancreatitis. Surg Clin North Am, 1989, 69(3):467-480.

20. Karne S, Gorelick FS - Etiopathogenesis of acute pancreatitis. Surg Clin North Am, 1999, 79(4):699-710.

21. Dernevis CG - Staging acute pancreatitis: where are we now? Pancreatology, 2001, 1(3):201-206.
22. Steer ML, Meldolesi J, Figarella C - Pancreatitis. The role of lysosomes. Dig Dis Sci, 1984, 29(10):934-938.

23. Saluja A, Hashimoto S, Saluja M, et al. - Subcellular redistribution of lysosomal enzymes during caeruleininduced pancreatitis. Am J Physiol, 1987, 253(4pt1):G508516.

24. Steer ML, Saluja AK - Lysosomal enzymes and pancreatitis. Gastroenterology, 1996, 110(3):965-967.

25. Meirelles Jr RF, Ceneviva R, Caboclo JLF, et al. - Pancreatic capillary blood flow during caerulein-induced pancreatitis evaluated by a laser-doppler flowmeter in rats. Acta Cir Bras, 2003, 18(suppl.5):18-22.

26. Meirelles Jr RF, Ceneviva R, Caboclo JLF, et al. - Reactive oxygen species inactivation improves pancreatic capillary blood flow in caerulein-induced pancreatitis in rats. Acta Cir Bras, 2003, 18(suppl.5):29-33.

27. Windsor JA, Hammodat $\mathrm{H}$ - Metabolic Management of severe acute pancreatitis. World J Surg, 2000, 24(6):664-672.

28. Klöppel G - "Pathology of severe acute pancreatitis". In Bradley EL - Acute pancreatitis: diagnosis and therapy. New York, Raven Press, 1994, pp.35-45.

29. Zhang Q, Ni Q, Cai D, et al. - Mechanisms of multiple organ damages in acute necrotizing pancreatitis. Chin Med J, 2001, 114(7):738-742.

30. Weber CK, Adler G - From acinar cell damage to systemic inflammatory response: current concepts in pancreatitis. Pancreatology, 2001, 1(4):356-362.

31. Gomez-Cambronero LG, Sabater L, Pereda J, et al. - Role of cytokines and oxidative stress in the pathophysiology of the pancreatitis: therapeutical implications. Curr Drug Targets Inflamm Allergy, 2002, 1(4):393-403.

32. Teague BD, Morrison CP, Court FG, et al. - The lack of a systemic inflammatory response syndrome supports the safety of pancreatic electrolysis: experimental studies. J Surg Res, 2004, 116(1):121-123.

33. Cunha Neto SH, Freire ECS, Magalhães ACG - "Pancreatite aguda". In Martins S, Souto MID - Manual de emergências médicas. $2^{\text {a }}$ Edição. Rio de Janeiro. Revinter, 2004, pp. 361366.

34. Bhatia M, Moochhala S - Role of inflammatory mediators in the pathophysiology of acute distress syndrome. J Pathol, 2004, 202(2):145-156

35. Liu HS, Pan CE, Liu QG, et al. - Effect of NF-kappaB and p38 MAPK in activated monocytes/macrophages on proinflammatory cytokines of rats with acute pancreatitis. World $\mathbf{J}$ Gastroenterol, 2003, 9(11):2513-2518.

36. Chen X, Ji B, Han B, et al. - NF-kappaB activation in pancreas induces pancreatic and systemic inflammatory response. Gastroenterology, 2002, 122(2):448-457.

37. Kylanpaa-Back ML, Takala A, Kemppainen E, et al. - Cellular markers of systemic inflammation and immune suppression in patients wit organ failure due to severe acute pancreatitis. Scand J Gastroenterol, 2001, 36(10):1100-1107.

38. Beger HG, Kunz R, Bittner R - "Prognostic criteria in necrotizing pancreatitis". In Beger HG, Bücher M - Acute pancreatitis. Berlin. Springer Verlag, 1987, pp. 198-200.

39. Beger HG, Rau B, Mayer J, et al. - Natural course of acute pancreatitis. World J Surg, 1997, 21(2):130-135.

40. Maggioni LCR, Nacif MS, Mota-Leal-Filho JM, et al. - "Infecções intra-abdominais" In Siqueira Batista R, Gomes AP, Santos SS, et al. (eds) - Manual de infectologia. Rio de Janeiro. Revinter; 2003, pp. 103-109.

41. Tavares W - Manual de antibióticos e quimioterápicos antiinfecciosos. $3^{\text {a }}$ Edição. São Paulo. Atheneu, 2001, pp. 529546 
42. Hotchkiss RS, Karl IE - The pathophysiology and treatment of sepsis. N Engl J Med, 2003, 348 (2):138-150.

43. Siqueira-Batista R, Gomes AP, Chamié D, et al. - "Sepse". In Siqueira Batista R, Gomes AP, Santos SS, et al. (eds) - Manual de infectologia. Rio de Janeiro. Revinter, 2003, pp. 158-162.

44. Siqueira-Batista R - Deuses e homens. Mito, filosofia e medicina na Grécia Antiga. São Paulo. Landy, 2003, pp. 217-228.

45. Hipócrates - Aforismos. Comentários de Carlos Brunini. São Paulo. Typus, 1998, pp. 112.
Endereço para correspondência:

Prof. Rodrigo Siqueira-Batista

Fundação Educacional Serra dos Órgãos (FESO)

Núcleo de Estudos em Filosofia e Saúde (NEFISA) - DPPE

Av. Alberto Torres, 111 - Alto. 25964-000 - Teresópolis - RJ

E-mail: anaximandro@hotmail.com 